\title{
Technical note: Dosimetric study of a new Co-60 source used in brachytherapy
}

\author{
D. Granero and J. Pérez-Calatayud \\ Physics Section, Radiation Oncology Department, "La Fe" University Hospital, Avenida Campanar 21, \\ E46009 Valencia, Spain \\ F. Ballester ${ }^{\text {a) }}$ \\ Department of Atomic, Molecular and Nuclear Physics and IFIC, University of Valencia-CSIC, \\ Dr. Moliner 50, E46100 Burjassot, Spain
}

(Received 3 April 2007; revised 22 May 2007; accepted for publication 20 June 2007; published 9 August 2007)

\begin{abstract}
The purpose of this study is to obtain the dosimetric parameters of a new Co-60 source used in high dose rate brachytherapy and manufactured by BEBIG (Eckert \& Ziegler BEBIG GmbH, Germany). The Monte Carlo method has been used to obtain the dose rate distribution in the updated TG-43U1 formalism of the American Association of Physicists in Medicine. In addition, to aid the quality control process on treatment planning systems (TPS), a two-dimensional rectangular dose rate table, coherent with the TG-43U1 dose calculation formalism, is given. These dosimetric data sets can be used as input data of the TPS calculations and to validate them. (C) 2007 American Association of Physicists in Medicine. [DOI: 10.1118/1.2759602]
\end{abstract}

Key words: brachytherapy, Co-60, Monte Carlo, HDR

\section{INTRODUCTION}

Although not as widespread as Ir-192, Co-60 is also available on afterloading equipment dedicated to high dose rate (HDR) brachytherapy, mainly addressed to the treatment of gynecological lesions. ${ }^{1-3}$

This study is aimed at obtaining the dose rate distribution in the TG-43 U1 formalism of the American Association of Physicists in Medicine (AAPM), ${ }^{4,5}$ and the 2D dose rate table in Cartesian coordinates of the new BEBIG (Eckert \& Ziegler BEBIG GmbH, Germany) ${ }^{60} \mathrm{Co}$ HDR source (model Co0.A86) using the Monte Carlo (MC) code GEAnT4. ${ }^{6}$ This new Co-60 source is a modified version of the old Co-60 source (model GK60M21) from BEBIG. ${ }^{7}$ The dosimetric data sets given in this study can be used as input and to validate the TPS calculations.

\section{MATERIAL AND METHODS}

The geometric design and materials of the new BEBIG Co-60 source (model Co0.A86) were obtained from the manufacturer and are shown schematically in Fig. 1. The new BEBIG ${ }^{60} \mathrm{Co}$ brachytherapy source is very similar to the old BEBIG source (model GK60M21), both in design and materials. The new source differs from the old one in that it has a smaller active core $(0.5 \mathrm{~mm}$ in diameter for the new source vs $0.6 \mathrm{~mm}$ in diameter for the old one) and a more rounded capsule tip. ${ }^{7}$ The new source is composed of a central cylindrical active core made of metallic ${ }^{60} \mathrm{Co}, 3.5 \mathrm{~mm}$ in length and with a diameter of $0.5 \mathrm{~mm}$. The active core is covered by a cylindrical stainless-steel capsule $0.15 \mathrm{~mm}$ thick with an external diameter of $1 \mathrm{~mm}$.

In order to obtain the dose rate distribution of the new ${ }^{60}$ Co source the Monte Carlo code GEANT4 (Ref. 6) (version 7.1) has been used. This code has been widely used by our group in other dosimetric studies of brachytherapy sources. ${ }^{7-9}$ The Monte Carlo code GEANT4 used in this study fulfills all the recommendations of the report, "Dosimetric prerequisites for routine clinical use of photon emitting brachytherapy sources with average energy higher than 50 keV," of the AAPM-ESTRO associations. ${ }^{10}$

The physics "low energy" models of GEANT4 have been used. These physics models use the EPDL97 cross-sections library ${ }^{11}$ for photons and the EEDL library ${ }^{11}$ for electrons. In order to speed up the simulations only the gamma part of the ${ }^{60} \mathrm{Co}$ spectrum was used. It has been verified that the contribution of the $\beta$ spectrum and electron spectra to the dose is negligible due to the presence of the stainless-steel cover around the metallic ${ }^{60} \mathrm{Co}$ where the electrons are stopped. ${ }^{7}$ The gamma spectrum used in the simulations was obtained from the NuDat database. ${ }^{12}$ A cutoff energy of $10 \mathrm{keV}$ was used for both photons and electrons. The methodology used for this Monte Carlo study follows the recommendations of the TG-43 U1 report. ${ }^{5}$

In order to obtain the dose rate distribution in water, the source has been located in the center of a spherical water phantom of $50 \mathrm{~cm}$ in radius that acts as an unbounded phantom for a ${ }^{60} \mathrm{Co}$ source up to a distance of $20 \mathrm{~cm}$ from the source. $^{3,7}$ The density used for the liquid water has been $0.998 \mathrm{~g} \mathrm{~cm}^{-3}$ at $22{ }^{\circ} \mathrm{C}$ as recommended in the TG-43 U1. To obtain the along and away dose rate table, a grid system composed of $400 \times 800$ cylindrical rings $0.05 \mathrm{~cm}$ thick and $0.05 \mathrm{~cm}$ high, concentric to the longitudinal source axis, was used, and to obtain the dose rate distribution in the form given by the TG43 report a system of $400 \times 180$ concentric spherical sections $0.05 \mathrm{~cm}$ thick with an angular width of $1^{\circ}$ in the polar angle $\theta$ was used. The coordinate axes used are shown in Fig. 1. 


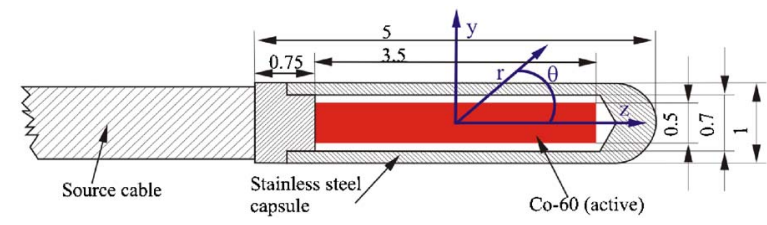

FIG. 1. Schematic view of the ${ }^{60}$ Co source (model Co0.A86). The coordinate axes used in this study are also shown with their origin situated in the geometric center of the active volume. Dimensions are in millimeters.

As electronic equilibrium conditions are not fulfilled in the near region of the source, both kerma and dose have been scored using the grid systems defined above. At points where electronic disequilibrium exists ${ }^{7}$ (those located at distances of less than $1 \mathrm{~cm}$ from the source) the values obtained as scoring dose are used to give the dose rate distribution, and at the points where electronic equilibrium exists the scored kerma values have been used to approximate dose because in this case kerma and dose match each other and the kerma is obtained with lower uncertainty. In an effort to speed up calculations and to reduce statistical uncertainties, kerma has been obtained using the linear track-length kerma estimator. $^{13}$

To estimate the air-kerma strength the BEBIG ${ }^{60} \mathrm{Co}$ source was located in the center of a $4 \times 4 \times 4 \mathrm{~m}^{3}$ air cube and kerma was scored using cylindrical ring cells, $1 \mathrm{~cm}$ thick and $1 \mathrm{~cm}$ high, located along the transverse source axis. The procedure used to extract the air-kerma strength is the same as that used in previous studies by our group. ${ }^{7}$

The number of photon histories simulated was $10^{9}$ to obtain kerma and $6 \times 10^{9}$ to obtain dose; $10^{8}$ photon histories have been simulated to obtain the air-kerma strength.

\section{RESULTS AND DISCUSSION}

The raw data obtained from the Monte Carlo simulations were analyzed with the ROOT data analysis framework ${ }^{14}$ in order to obtain the along-away 2D Cartesian lookup data $\dot{D}(y, z)$ and the TG-43 dosimetric parameters.

TABLE I. Dose rate in an unbounded liquid water phantom per unit air-kerma strength (cGy h${ }^{-1} \mathrm{U}^{-1}$ ) around the new BEBIG ${ }^{60} \mathrm{Co}$ source (model Co0.A86). The coordinate axes are defined in Fig. 1.

Distance away $y(\mathrm{~cm})$

\begin{tabular}{|c|c|c|c|c|c|c|c|c|c|c|c|c|c|c|c|}
\hline $\begin{array}{l}\text { Distance } \\
\text { along } \\
z \\
(\mathrm{~cm})\end{array}$ & 0 & 0.25 & 0.5 & 0.75 & 1 & 1.5 & 2 & 2.5 & 3 & 4 & 5 & 6 & 8 & 10 & 14 \\
\hline-14 & 0.00399 & 0.00409 & 0.00404 & 0.00405 & 0.00407 & 0.00408 & 0.00406 & 0.00403 & 0.00398 & 0.00386 & 0.00370 & 0.00350 & 0.00308 & 0.00263 & 0.00185 \\
\hline-10 & 0.00853 & 0.00866 & 0.00867 & 0.00867 & 0.00870 & 0.00870 & 0.00861 & 0.00847 & 0.00828 & 0.00778 & 0.00718 & 0.00655 & 0.00531 & 0.00423 & 0.00264 \\
\hline-8 & 0.01400 & 0.01401 & 0.01409 & 0.01414 & 0.01418 & 0.01406 & 0.01380 & 0.01342 & 0.01294 & 0.01177 & 0.01052 & 0.00927 & 0.00705 & 0.00533 & 0.00310 \\
\hline-6 & 0.0258 & 0.0259 & 0.0260 & 0.0261 & 0.0260 & 0.0255 & 0.0246 & 0.0233 & 0.0219 & 0.01882 & 0.01589 & 0.01330 & 0.00931 & 0.00660 & 0.00355 \\
\hline-5 & 0.0380 & 0.0380 & 0.0382 & 0.0382 & 0.0379 & 0.0366 & 0.0346 & 0.0321 & 0.0295 & 0.0243 & 0.01970 & 0.01595 & 0.01059 & 0.00726 & 0.00377 \\
\hline-4 & 0.0602 & 0.0602 & 0.0608 & 0.0604 & 0.0596 & 0.0560 & 0.0513 & 0.0460 & 0.0408 & 0.0316 & 0.0244 & 0.01894 & 0.01190 & 0.00789 & 0.00396 \\
\hline-3 & 0.1077 & 0.1092 & 0.1096 & 0.1078 & 0.1040 & 0.0930 & 0.0804 & 0.0684 & 0.0577 & 0.0410 & 0.0297 & 0.0221 & 0.01315 & 0.00847 & 0.00412 \\
\hline-2.5 & 0.1562 & 0.1591 & 0.1585 & 0.1535 & 0.1453 & 0.1245 & 0.1030 & 0.0840 & 0.0685 & 0.0463 & 0.0325 & 0.0236 & 0.01371 & 0.00871 & 0.00418 \\
\hline-2 & 0.247 & 0.252 & 0.247 & 0.233 & 0.214 & 0.1708 & 0.1329 & 0.1032 & 0.0808 & 0.0518 & 0.0352 & 0.0251 & 0.01420 & 0.0089 & 0.0042 \\
\hline-1.5 & 0.447 & 0.453 & 0.430 & 0.384 & 0.333 & 0.239 & 0.1713 & 0.1251 & 0.0938 & 0.0570 & 0.0375 & 0.0263 & 0.01461 & 0.00909 & 0.00428 \\
\hline-1 & 1.035 & 1.026 & 0.882 & 0.703 & 0.546 & 0.333 & 0.215 & 0.1472 & 0.1059 & 0.0613 & 0.0394 & 0.0272 & 0.01493 & 0.00921 & 0.00431 \\
\hline-0.75 & $\cdots$ & 1.806 & 1.384 & 0.985 & 0.701 & 0.386 & 0.236 & 0.1569 & 0.1109 & 0.0630 & 0.0401 & 0.0276 & 0.01503 & 0.00926 & 0.00432 \\
\hline-0.5 & $\cdots$ & 3.82 & 2.27 & 1.365 & 0.874 & 0.434 & 0.254 & 0.1645 & 0.1147 & 0.0642 & 0.0406 & 0.0278 & 0.01511 & 0.00929 & 0.00433 \\
\hline-0.25 & $\cdots$ & 9.47 & 3.57 & 1.763 & 1.025 & 0.469 & 0.265 & 0.1695 & 0.1172 & 0.0650 & 0.0410 & 0.0280 & 0.01514 & 0.00931 & 0.00433 \\
\hline 0 & $\cdots$ & 15.15 & 4.32 & 1.929 & 1.087 & 0.482 & 0.269 & 0.1712 & 0.1179 & 0.0652 & 0.0410 & 0.0280 & 0.01516 & 0.00931 & 0.00434 \\
\hline 0.25 & $\cdots$ & 9.48 & 3.58 & 1.758 & 1.025 & 0.469 & 0.265 & 0.1694 & 0.1171 & 0.0650 & 0.0410 & 0.0280 & 0.01516 & 0.00932 & 0.00434 \\
\hline 0.5 & 4.83 & 3.86 & 2.26 & 1.368 & 0.874 & 0.434 & 0.253 & 0.1645 & 0.1147 & 0.0642 & 0.0406 & 0.0278 & 0.01511 & 0.00930 & 0.00433 \\
\hline 0.75 & 2.02 & 1.829 & 1.389 & 0.986 & 0.700 & 0.385 & 0.236 & 0.1568 & 0.1109 & 0.0630 & 0.0401 & 0.0276 & 0.01503 & 0.00926 & 0.00432 \\
\hline 1 & 1.079 & 1.037 & 0.884 & 0.703 & 0.546 & 0.333 & 0.215 & 0.1472 & 0.1058 & 0.0613 & 0.0394 & 0.0272 & 0.01493 & 0.00922 & 0.00431 \\
\hline 1.5 & 0.466 & 0.463 & 0.432 & 0.385 & 0.333 & 0.239 & 0.1713 & 0.1251 & 0.0938 & 0.0570 & 0.0375 & 0.0263 & 0.01461 & 0.00909 & 0.00428 \\
\hline 2 & 0.258 & 0.259 & 0.250 & 0.234 & 0.214 & 0.1710 & 0.1329 & 0.1032 & 0.0808 & 0.0517 & 0.0352 & 0.0250 & 0.01420 & 0.00892 & 0.00424 \\
\hline 2.5 & 0.1631 & 0.1647 & 0.1610 & 0.1546 & 0.1459 & 0.1245 & 0.1030 & 0.0840 & 0.0685 & 0.0463 & 0.0325 & 0.0237 & 0.01371 & 0.00871 & 0.00418 \\
\hline 3 & 0.1123 & 0.1132 & 0.1118 & 0.1088 & 0.1044 & 0.0932 & 0.0806 & 0.0685 & 0.0577 & 0.0410 & 0.0297 & 0.0221 & 0.01315 & 0.00847 & 0.00412 \\
\hline 4 & 0.0618 & 0.0626 & 0.0623 & 0.0615 & 0.0601 & 0.0563 & 0.0514 & 0.0461 & 0.0409 & 0.0316 & 0.0244 & 0.01893 & 0.01190 & 0.00790 & 0.00395 \\
\hline 5 & 0.0390 & 0.0393 & 0.0393 & 0.0390 & 0.0385 & 0.0369 & 0.0347 & 0.0322 & 0.0296 & 0.0243 & 0.01972 & 0.01594 & 0.01058 & 0.00726 & 0.00377 \\
\hline 6 & 0.0266 & 0.0268 & 0.0269 & 0.0268 & 0.0265 & 0.0258 & 0.0247 & 0.0234 & 0.0219 & 0.01887 & 0.01592 & 0.01331 & 0.00930 & 0.00660 & 0.00356 \\
\hline 8 & 0.01454 & 0.01460 & 0.01455 & 0.01456 & 0.01449 & 0.01428 & 0.01394 & 0.01348 & 0.01298 & 0.01181 & 0.01052 & 0.00927 & 0.00705 & 0.00533 & 0.00310 \\
\hline 10 & 0.00884 & 0.00899 & 0.00895 & 0.00896 & 0.00893 & 0.00885 & 0.00871 & 0.00856 & 0.00833 & 0.00780 & 0.00720 & 0.00656 & 0.00531 & 0.00423 & 0.00265 \\
\hline 14 & 0.00414 & 0.00418 & 0.00418 & 0.00419 & 0.00419 & 0.00416 & 0.00412 & 0.00409 & 0.00403 & 0.00389 & 0.00372 & 0.00352 & 0.00308 & 0.00263 & 0.00185 \\
\hline
\end{tabular}


TABLE II. Anisotropy function $F(r, \theta)$ for the new BEBIG ${ }^{60}$ Co source (model Co0.A86). The coordinate axes are defined in Fig. 1.

\begin{tabular}{|c|c|c|c|c|c|c|c|c|c|c|c|c|c|c|c|c|}
\hline \multirow[b]{2}{*}{$\theta$ (degrees) } & \multicolumn{16}{|c|}{$r(\mathrm{~cm})$} \\
\hline & 0.25 & 0.5 & .75 & 1 & 1.5 & 2 & 3 & 4 & 5 & 6 & 7 & 8 & 10 & 12 & 15 & 20 \\
\hline 0 & $\cdots$ & 0.939 & 0.966 & 0.945 & 0.945 & 0.947 & 0.945 & 0.944 & 0.945 & 0.945 & 0.947 & 0.947 & 0.952 & 0.955 & 0.958 & 0.959 \\
\hline 1 & $\cdots$ & 0.941 & 0.969 & 0.948 & 0.947 & 0.947 & 0.946 & 0.946 & 0.948 & 0.949 & 0.951 & 0.952 & 0.955 & 0.957 & 0.961 & 0.962 \\
\hline 2 & $\cdots$ & 0.938 & 0.968 & 0.952 & 0.952 & 0.952 & 0.952 & 0.953 & 0.955 & 0.956 & 0.957 & 0.959 & 0.959 & 0.962 & 0.964 & 0.966 \\
\hline 3 & $\cdots$ & 0.939 & 0.969 & 0.956 & 0.956 & 0.957 & 0.958 & 0.958 & 0.959 & 0.960 & 0.961 & 0.962 & 0.964 & 0.965 & 0.967 & 0.970 \\
\hline 4 & $\cdots$ & 0.941 & 0.972 & 0.959 & 0.959 & 0.960 & 0.961 & 0.962 & 0.962 & 0.964 & 0.965 & 0.966 & 0.968 & 0.968 & 0.970 & 0.975 \\
\hline 5 & $\cdots$ & 0.944 & 0.975 & 0.962 & 0.962 & 0.962 & 0.963 & 0.965 & 0.965 & 0.966 & 0.967 & 0.968 & 0.970 & 0.970 & 0.971 & 0.976 \\
\hline 6 & $\cdots$ & 0.947 & 0.979 & 0.964 & 0.964 & 0.964 & 0.964 & 0.965 & 0.965 & 0.966 & 0.967 & 0.968 & 0.970 & 0.970 & 0.972 & 0.975 \\
\hline 8 & $\cdots$ & 0.954 & 0.980 & 0.968 & 0.967 & 0.967 & 0.967 & 0.968 & 0.968 & 0.969 & 0.970 & 0.971 & 0.972 & 0.973 & 0.975 & 0.975 \\
\hline 10 & $\cdots$ & 0.957 & 0.980 & 0.971 & 0.971 & 0.971 & 0.971 & 0.972 & 0.972 & 0.973 & 0.973 & 0.974 & 0.975 & 0.975 & 0.977 & 0.979 \\
\hline 15 & $\cdots$ & 0.961 & 0.981 & 0.978 & 0.979 & 0.979 & 0.979 & 0.979 & 0.979 & 0.980 & 0.980 & 0.980 & 0.981 & 0.981 & 0.982 & 0.983 \\
\hline 20 & $\cdots$ & 0.972 & 0.990 & 0.985 & 0.985 & 0.985 & 0.985 & 0.985 & 0.985 & 0.985 & 0.986 & 0.986 & 0.986 & 0.986 & 0.986 & 0.987 \\
\hline 25 & $\cdots$ & 0.987 & 0.989 & 0.988 & 0.989 & 0.989 & 0.989 & 0.989 & 0.989 & 0.990 & 0.990 & 0.990 & 0.990 & 0.990 & 0.990 & 0.991 \\
\hline 30 & $\cdots$ & 0.991 & 0.996 & 0.993 & 0.993 & 0.993 & 0.993 & 0.993 & 0.992 & 0.993 & 0.993 & 0.993 & 0.992 & 0.993 & 0.992 & 0.993 \\
\hline 40 & $\cdots$ & 0.998 & 0.998 & 0.996 & 0.996 & 0.996 & 0.996 & 0.996 & 0.996 & 0.996 & 0.996 & 0.996 & 0.996 & 0.996 & 0.996 & 0.997 \\
\hline 50 & $\cdots$ & 1.000 & 1.000 & 0.998 & 0.998 & 0.998 & 0.998 & 0.998 & 0.998 & 0.998 & 0.998 & 0.998 & 0.999 & 0.998 & 0.998 & 0.998 \\
\hline 60 & 0.957 & 0.999 & 1.002 & 0.999 & 0.999 & 0.999 & 0.999 & 0.999 & 0.998 & 0.998 & 0.999 & 0.999 & 0.999 & 0.998 & 0.998 & 0.999 \\
\hline 70 & 0.974 & 0.998 & 1.001 & 1.000 & 1.000 & 1.000 & 1.000 & 1.000 & 0.999 & 1.000 & 1.000 & 1.000 & 1.000 & 0.999 & 0.999 & 1.000 \\
\hline 80 & 0.990 & 0.994 & 0.999 & 1.000 & 1.000 & 1.000 & 1.000 & 1.000 & 1.000 & 1.000 & 1.000 & 1.000 & 1.000 & 0.999 & 1.000 & 1.000 \\
\hline 90 & 1 & 1 & 1 & 1 & 1 & 1 & 1 & 1 & 1 & 1 & 1 & 1 & 1 & 1 & 1 & 1 \\
\hline 100 & 0.990 & 0.994 & 1.000 & 1.001 & 1.000 & 1.000 & 1.000 & 1.000 & 1.000 & 1.000 & 1.000 & 1.000 & 1.000 & 0.999 & 1.000 & 1.000 \\
\hline 110 & 0.975 & 0.997 & 1.002 & 1.000 & 1.000 & 1.000 & 1.000 & 1.000 & 0.999 & 0.999 & 1.000 & 0.999 & 1.000 & 0.999 & 0.999 & 1.000 \\
\hline 120 & 0.956 & 0.999 & 1.000 & 0.999 & 0.999 & 0.999 & 0.999 & 0.999 & 0.998 & 0.998 & 0.998 & 0.999 & 0.998 & 0.998 & 0.998 & 0.999 \\
\hline 130 & $\cdots$ & 1.000 & 1.001 & 0.997 & 0.999 & 0.999 & 0.998 & 0.998 & 0.998 & 0.997 & 0.998 & 0.998 & 0.998 & 0.998 & 0.997 & 0.997 \\
\hline 140 & $\cdots$ & 0.994 & 0.996 & 0.995 & 0.996 & 0.995 & 0.995 & 0.995 & 0.995 & 0.995 & 0.995 & 0.995 & 0.995 & 0.995 & 0.995 & 0.995 \\
\hline 150 & $\cdots$ & 0.983 & 0.996 & 0.991 & 0.991 & 0.991 & 0.990 & 0.990 & 0.990 & 0.990 & 0.991 & 0.991 & 0.991 & 0.991 & 0.991 & 0.991 \\
\hline 155 & $\cdots$ & $\cdots$ & 0.985 & 0.987 & 0.987 & 0.986 & 0.986 & 0.987 & 0.986 & 0.986 & 0.987 & 0.987 & 0.987 & 0.987 & 0.988 & 0.988 \\
\hline 160 & $\ldots$ & $\ldots$ & 0.976 & 0.980 & 0.980 & 0.981 & 0.981 & 0.981 & 0.981 & 0.981 & 0.982 & 0.982 & 0.982 & 0.982 & 0.983 & 0.984 \\
\hline 165 & $\ldots$ & $\cdots$ & $\cdots$ & 0.970 & 0.969 & 0.969 & 0.969 & 0.970 & 0.970 & 0.970 & 0.971 & 0.972 & 0.973 & 0.973 & 0.974 & 0.976 \\
\hline 170 & $\cdots$ & $\cdots$ & $\cdots$ & 0.953 & 0.954 & 0.954 & 0.955 & 0.956 & 0.957 & 0.958 & 0.960 & 0.961 & 0.962 & 0.963 & 0.965 & 0.968 \\
\hline 172 & $\cdots$ & $\cdots$ & $\cdots$ & 0.944 & 0.944 & 0.944 & 0.946 & 0.947 & 0.949 & 0.950 & 0.951 & 0.952 & 0.954 & 0.955 & 0.959 & 0.961 \\
\hline 174 & $\cdots$ & $\cdots$ & $\cdots$ & 0.934 & 0.934 & 0.934 & 0.936 & 0.937 & 0.939 & 0.941 & 0.942 & 0.944 & 0.947 & 0.948 & 0.951 & 0.956 \\
\hline 175 & $\ldots$ & $\ldots$ & $\ldots$ & 0.926 & 0.927 & 0.928 & 0.930 & 0.932 & 0.934 & 0.936 & 0.938 & 0.939 & 0.942 & 0.945 & 0.948 & 0.953 \\
\hline 176 & $\cdots$ & $\cdots$ & $\cdots$ & 0.921 & 0.922 & 0.923 & 0.925 & 0.928 & 0.929 & 0.931 & 0.934 & 0.936 & 0.939 & 0.943 & 0.947 & 0.952 \\
\hline 177 & $\cdots$ & $\cdots$ & $\cdots$ & 0.919 & 0.919 & 0.919 & 0.921 & 0.924 & 0.926 & 0.928 & 0.930 & 0.932 & 0.936 & 0.940 & 0.943 & 0.947 \\
\hline 178 & $\cdots$ & $\cdots$ & $\cdots$ & 0.913 & 0.913 & 0.914 & 0.918 & 0.920 & 0.922 & 0.924 & 0.925 & 0.929 & 0.933 & 0.936 & 0.939 & 0.944 \\
\hline 179 & $\cdots$ & $\cdots$ & $\cdots$ & 0.910 & 0.908 & 0.908 & 0.911 & 0.913 & 0.915 & 0.918 & 0.918 & 0.921 & 0.926 & 0.930 & 0.934 & 0.938 \\
\hline
\end{tabular}

The along-away 2D Cartesian lookup data $\dot{D}(y, z)$ are shown in Table I. To obtain the TG-43U1 dosimetric parameters from $\dot{D}(r, \theta)$ we have used the geometric factor $G_{L}(r, \theta)$ with a length of $L=3.5 \mathrm{~mm}$. The dose rate constant obtained has been $\Lambda=1.087 \pm 0.011 \mathrm{cGy} \mathrm{h}^{-1} \mathrm{U}^{-1}$, where the air-kerma strength is expressed in units $1 \mathrm{U}=1 \mu \mathrm{Gyh}^{-1} \mathrm{~m}^{2}$. The anisotropy function, $F(r, \theta)$, is presented in Table II and the radial dose function, $g_{L}(r)$, in Table III.

The uncertainties in the final dose rate distributions for ${ }^{60} \mathrm{Co}$ sources can be reduced to only the statistical uncertainties: ${ }^{7}$ thus, in this study the final uncertainty (standard deviation of the mean with $k=1$ ) is less than $0.7 \%$ for all the points, except at the points located near the longitudinal axis, where it is about $1.1 \%$.

The dose rate distribution obtained for the new BEBIG source has been compared with that obtained for the old BEBIG HDR source ${ }^{7}$ in Fig. 2. This comparison shows that both dose rate distributions are nearly identical in front of the source, the differences between both sources less being than $0.5 \%$. In the zone near the longitudinal source axis, they are less than $4 \%$ for $z>0$ and less than $10 \%$ for $z<0$. In the latter case the differences are due to the fact that, in the study of the old BEBIG HDR source, the source cable length was $20 \mathrm{~mm}$, and in this study it was $5 \mathrm{~mm}$, which reproduces the clinical setup better on highly curved catheters.

\section{CONCLUSIONS}

In this study, a complete dosimetric data set for the new BEBIG ${ }^{60} \mathrm{Co}$ source (model Co0.A86) was obtained for an unbounded liquid water phantom using the Monte Carlo GEANT4 code. Functions and parameters following TG-43U1 formalism are presented: the dose rate constant, the radial dose function, and the anisotropy function. In addition, in order to aid quality control on TPS, a 2D rectangular dose 
TABLE III. Radial dose function for the BEBIG ${ }^{60} \mathrm{Co}$ source (model Co0.A86). The radial dose function has been fitted to a third-order polynomial with coefficients $a_{0}=1.0159, a_{1}=-1.577 \times 10^{-2}, a_{2}=-1.020 \times 10^{-4}$, $a_{3}=-2.404 \times 10^{-7}$ between 0.25 and $20 \mathrm{~cm}$. Note that the fit is the same that used in for the old BEBIG Co-60 source (Ref. 7).

\begin{tabular}{cl}
\hline \hline Distance $r(\mathrm{~cm})$ & $g_{L}(r)$ \\
\hline 0.25 & 1.007 \\
0.5 & 1.036 \\
0.75 & 1.015 \\
1 & 1 \\
1.5 & 0.992 \\
2 & 0.984 \\
3 & 0.968 \\
4 & 0.952 \\
5 & 0.936 \\
6 & 0.919 \\
7 & 0.902 \\
8 & 0.884 \\
10 & 0.849 \\
12 & 0.813 \\
15 & 0.756 \\
20 & 0.665 \\
\hline \hline
\end{tabular}

rate table consistent with the TG43 dose calculation formalism is given.

\section{ACKNOWLEDGMENTS}

The authors acknowledge Eckert \& Ziegler BEBIG $\mathrm{GmbH}$, Germany, who provided the technical details of sources. This study was supported in part by the Ministerio de Educación y Ciencia (Spain): Project Nos. FPA200612120-C03-02, DPI2004-04268-C02-01, and FIS200405713; European Community Project No. FP6-2005LIFESCIHEALTH-7 FP6-037555. The authors acknowledge the Fundación IVO (Valencia, Spain) for its research support.

\footnotetext{
${ }^{a)}$ Electronic mail: facundo.ballester@uv.es

${ }^{1} \mathrm{H}$. Ito, "Radiotherapy for cervical cancer: A multi-institutional comparison," Nippon Igaku Hoshasen Gakkai Zasshi 59, 745-749 (1999).

${ }^{2}$ W. C. Kim, G. E. Kim, C. O. Suh, and J. J. K. Loh, "High versus low dose rate intracavitary irradiation for adenocarcinoma of the uterine cervix," Jpn. J. Clin. Oncol. 31, 432-437 (2001).

${ }^{3}$ P. Papagiannis, A. Angelopoulos, E. vPantelis, L. Sakelliou, P. Karaiskos, and Y. Shimizu, "Monte Carlo dosimetry of ${ }^{60} \mathrm{Co}$ HDR brachytherapy sources," Med. Phys. 30, 712-721 (2003).

${ }^{4}$ R. Nath, L. L. Anderson, G. Luxton, K. A. Weaver, J. F. Williamson, and A. S. Meigooni, "Dosimetry of interstitial brachytherapy sources: Recommendations of the AAPM Radiation Therapy Committee Task Group No. 43," Med. Phys. 22, 209-234 (1995).

${ }^{5}$ M. J. Rivard, B. M. Coursey, L. A. DeWerd, W. F. Hanson, M. S. Huq, G. S. Ibbott, M. J. Mitch, R. Nath, and J. F. Williamson, "Update of AAPM Task Group No. 43 report: A revised AAPM protocol for brachytherapy dose calculations," Med. Phys. 31, 633-674 (2004).

${ }^{6}$ S. Agostinelli et al., "GEANT4-A simulation toolkit," Nucl. Instrum. Methods Phys. Res. A 506, 250-303 (2003); (see also http:// geant4.cern.ch/, last accessed 2-19-2007).

${ }^{7}$ F. Ballester, D. Granero, J. Perez-Calatayud, E. Casal, S. Agramunt, and R. Cases, "Monte Carlo dosimetric study of the BEBIG Co-60 HDR source," Phys. Med. Biol. 50, N309-N316 (2005).
}

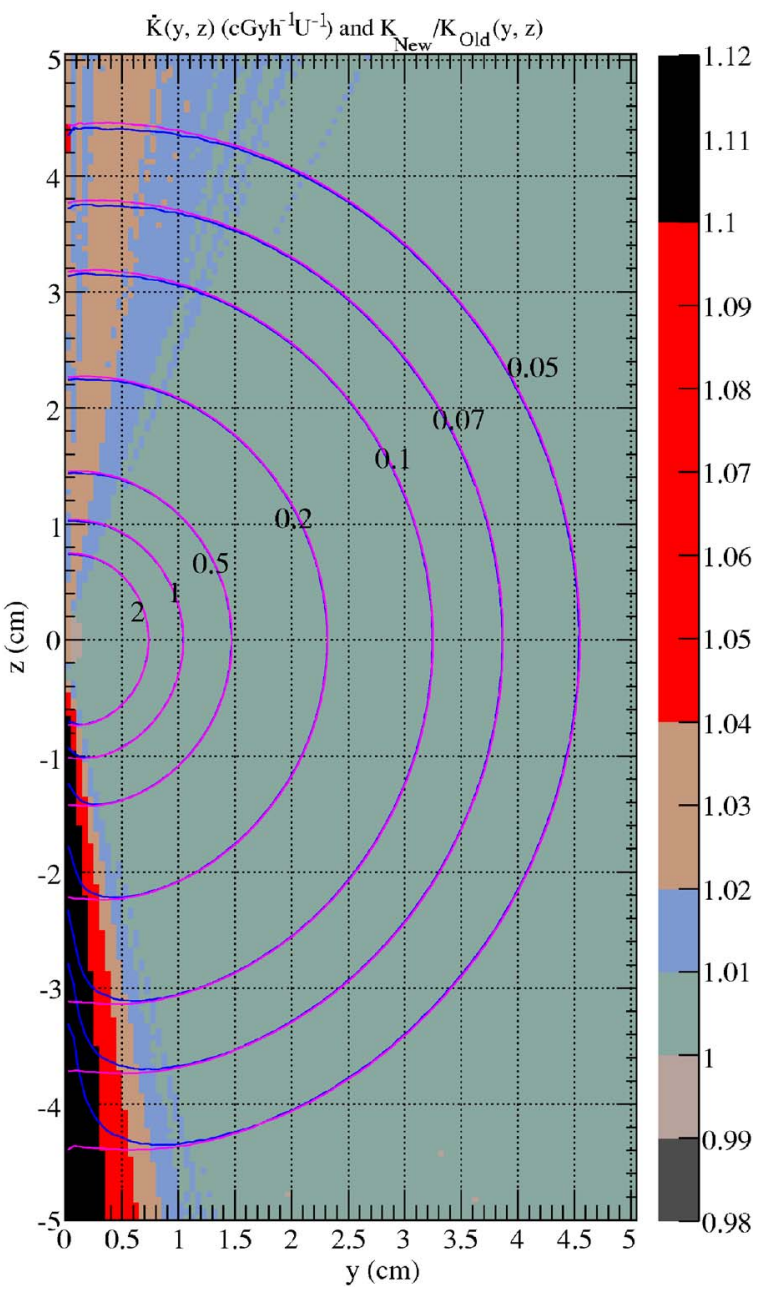

FIG. 2. Isodose curves in $\mathrm{cGyh}^{-1} \mathrm{U}^{-1}$ for the old BEBIG source and the new BEBIG HDR source. The background of the figure is the ratio between the kerma rate distribution obtained for the new and the old sources.

${ }^{8}$ J. Pérez-Calatayud, D. Granero, F. Ballester, V. Puchades, and E. Casal, "Monte Carlo dosimetric characterization of the Cs-137 selectron/LDR source: Evaluation of applicator attenuation and superposition approximation effects," Med. Phys. 31, 493-499 (2004).

${ }^{9}$ D. Granero, J. Pérez-Calatayud, and F. Ballester, "Monte Carlo calculation of TG-43 dosimetric parameters of a new BEBIG Ir-192 HDR source," Radiother. Oncol. 76, 79-85 (2005).

${ }^{10}$ Z. Li, R. K. Das, L. A. DeWerd, G. S. Ibbot, A. S. Meigooni, J. PérezCalatayud, M. J. Rivard, R. S. Sloboda, and J. F. Williamson, "Dosimetric prerequisites for routine clinical use of photon emitting brachytherapy sources with average energy higher than $50 \mathrm{keV}$," Med. Phys. 34, 37-40 (2007).

${ }^{11}$ D. E. Cullen, S. T. Perkins and S. M. Seltzer, "Tables and graphs of electron-interaction cross sections from $10 \mathrm{eV}$ to $100 \mathrm{GeV}$ derived from the LLNL Evaluated Electron Data Library (EEDL), $\quad Z=1-100$," Lawrence Livermore National Laboratory, UCRL-50400, Vol. 31 (2001). ${ }^{12}$ NuDat 2.0 National Nuclear Data Center Nuclear data from NuDat, a web-based database maintained by the National Nuclear Data Center Brookhaven National Laboratory (Upton, NY) (2004); http:// www.nndc.bnl.gov/nudat2 (last accessed 2-12-2007).

${ }^{13}$ J. F. Williamson, "Monte Carlo evaluation of kerma at a point for photon transport problems," Med. Phys. 14, 567-576 (1987).

${ }^{14}$ R. Brun and F. Rademakers, "ROOT-An Object Oriented Data Analysis Framework," Nucl. Instrum. Methods Phys. Res. A 389, 81-86 (1997) ; (see also http://root.cern.ch/, last accessed 2-12-2007). 